\title{
A Rare Presentation of Leptospirosis
}

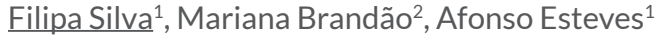 \\ ${ }^{1}$ Internal Medicine Department, Centro Hospitala de São João, Porto, Portugal \\ ${ }^{2}$ Medical Oncology Department, Instituto Português de Oncologia, Porto, Portugal
}

Received: 07/06/2016

Accepted: 22/07/2016

Published: $26 / 08 / 2016$

How to cite this article: Silva F, Brandão M, Esteves A. A rare presentation of leptospirosis. EJCRIM 2016;3:doi:10.12890/2016_000447.

Conflicts of Interests: The Authors declare that there are no competing interests.

This article is licensed under a Commons Attribution Non-Commercial 4.0 License

\section{ABSTRACT}

Leptospirosis has a wide spectrum of clinical manifestations. Acute renal failure, an important complication, generally involves interstitial and tubular damage. We describe the case of a 42-year-old man who was admitted with fever, back pain and periorbital oedema. He had hypertension, thrombocytopenia, acute renal failure, hypoalbuminaemia, hypertriglyceridaemia and proteinuria $>4.00 \mathrm{~g} / \mathrm{l}$. The renal biopsy showed mesangioproliferative glomerulonephritis. Due to the epidemiological context and clinical picture, ceftriaxone was started with rapid clinical improvement. Blood PCR for leptospira came back positive. The presentation of leptospirosis as nephrotic syndrome is rare and this diagnosis should be considered before performing a renal biopsy.

\section{LEARNING POINTS}

- Leptospirosis is a zoonosis with a wide spectrum of clinical manifestations including acute renal failure, which is an important complication.

- Leptospirosis should be considered in a patient with nephrotic syndrome and acute renal failure if the epidemiological context is favourable.

- Leptospirosis is one of the causes of nephrotic syndrome where a renal biopsy is not indicated.

\section{KEYWORDS}

Leptospirosis; acute renal failure; nephrotic syndrome; renal biopsy

\section{INTRODUCTION}

Leptospirosis, a spirochaetal infection, is an important zoonosis with a worldwide distribution ${ }^{[1]}$. A broad spectrum of clinical manifestations may occur and can be divided into two distinct clinical syndromes: a mild anicteric febrile illness and a severe form of leptospirosis with jaundice and other manifestations (known as Weil's disease) ${ }^{[1,2]}$. The renal involvement in leptospirosis can have a subclinical course, with mild proteinuria and abnormal urinary sediments, or present as overt acute kidney injury (AKI). It is a frequent complication and mainly characterized by interstitial and tubular damage ${ }^{[2]}$. In this report we describe the case of a 42-year-old male patient who had an atypical and rare presentation of leptospirosis in the form of nephrotic syndrome. 


\section{CASE REPORT}

A 42-year-old Caucasian man, an employee of a construction company, was asymptomatic until 1 week before admission when he started to complaint of moderate but continuous lumbar pain associated with chills and fever up to $39^{\circ} \mathrm{C}$. He denied dysuria, haematuria or other urinary complaints. He was evaluated in the emergency department (ED) and the work-up revealed thrombocytopenia of $106 \times 10^{\circ} / \mathrm{I}$ with a normal white blood cell count and haemoglobin, normal renal and liver function, and urine analysis which showed proteinuria 3+ without leukocytes or blood. He was prescribed cefuroxime and discharged. However, the bilateral periorbital oedema and hypertension persisted so he returned to the ED. He denied arthralgia, cutaneous lesions, chest pain and dyspnoea.

Besides a smoking habit (22 pack-years) and a spontaneous pneumothorax 30 years previously, his past medical history was irrelevant, and he denied hypertension, diabetes mellitus, alcohol and illicit drug use. In terms of epidemiological risk, he denied direct contact with animals, but had been working for several months in contact with sewage.

On initial evaluation at ED his temperature was $38.5^{\circ} \mathrm{C}$, blood pressure was $168 / 100 \mathrm{mmHg}$, pulse 72 beats $/ \mathrm{min}$ and oxygen saturation $99 \%$ in ambient air. On physical examination he was conscious and anicteric, with periorbital and lower limb oedema. Pulmonary auscultation revealed reduced breath sounds over the lung bases bilaterally, there was no hepatosplenomegaly and he had a positive bilateral Murphy's kidney punch sign.

Workup revealed thrombocytopenia of 55,000 $\times 10^{9} / \mathrm{l}$, C-reactive protein of $61.5 \mathrm{mg} / \mathrm{l}$ (normal $<3 \mathrm{mg} / \mathrm{l}$ ), acute renal failure (creatinine 3.10 $\mathrm{mg} / \mathrm{dl})$, hypoalbuminaemia $(23.9 \mathrm{~g} / \mathrm{l})$, hypertriglyceridaemia $(267 \mathrm{mg} / \mathrm{dl}$ ) and proteinuria $\geq 4.00 \mathrm{~g} / \mathrm{l}$. Renal ultrasound showed hepatomegaly and enlarged hyperechogenic kidneys, and chest x-ray revealed bilateral pleural effusion.

An autoimmune study and serological tests for HIV and hepatitis were negative. A 24-hour urine collection confirmed proteinuria of 4.96 $\mathrm{g} / 24 \mathrm{~h}$. Due to the development of oliguria and worsening renal function, a renal biopsy was performed and showed mesangioproliferative glomerulonephritis (Fig. 1). At this time due to the epidemiological context and clinical picture, leptospirosis had already been considered and the patient was started on a 7-day course of ceftriaxone with clinical improvement, and decreasing creatinine and proteinuria until normalization. PCR for leptospira in blood came back positive.

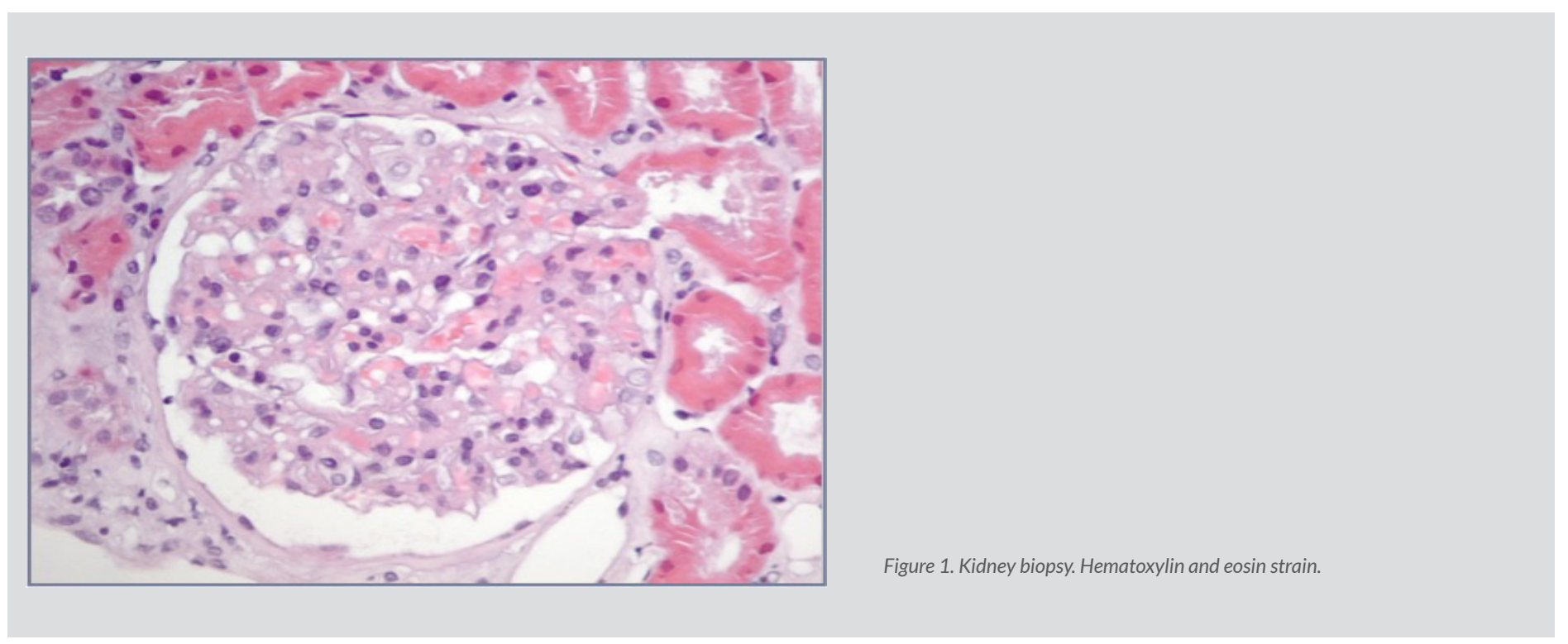

\section{DISCUSSION}

By discussing this clinical case, the authors wish to draw attention to this rare entity, since a delay in diagnosis can lead both to a chronic carrier state and to potentially irreversible renal injury, with attendant costs that could have be avoided with prompt institution of hydration and an appropriate antibiotic.

Leptospirosis is a disease with variable clinical manifestations and an incubation period of 2-26 days ${ }^{[3]}$. There are two distinct phases: septicaemia and the immune phase. The septicaemic phase is the result of vascular injury, while the immune phase is due to immune complex deposition leading to endothelial cell damage ${ }^{[1]}$. 
Kidney involvement was first reported in 1907, when Stimson described this microorganism in the renal tubules of a patient who died of a yellow fever ${ }^{[3]}$. The incidence of AKI varies from $10 \%$ to $60 \%$ depending on the severity of the disease, age, and definition used ${ }^{[1]}$. The typical picture is non-oliguric, hypokalaemic renal insufficiency, with impaired proximal sodium reabsorption, increased distal sodium delivery and potassium wasting. As volume depletion progresses, oliguric renal insufficiency can develop and lead to acute tubular necrosis ${ }^{[1,4,5]}$.

Although leptospirosis is an important cause of acute renal failure, the mechanisms of renal dysfunction have not been fully clarified. So far, the main factors implicated are the direct nephrotoxic action of the leptospira and the toxin-induced immune response. The outer membrane of leptospira contains antigenic components such as lipoproteins, lipopolysaccharides and peptidoglycans which function as endotoxins that can cause tubular dysfunction and inflammation. The most important outer membrane protein is LipL32, which directly affects the proximal tubular cells, increasing the expression of genes and pro-inflammatory proteins, such as nitric oxide synthase, monocyte chemotactic protein-1, T cells and tumour necrosis factor $\alpha$. Other causes are haemodynamic alterations, jaundice and rhabdomyolysis ${ }^{[1,2]}$. Renal biopsy is indicated in idiopathic nephrotic syndrome since the presence of focal segmental glomerulosclerosis, membranous nephropathy or minimal change disease may indicate the severity of the disease, prognosis and direct therapeutic interventions. However, there are some causes of nephrotic syndrome where a renal biopsy is not indicated. The more common are long-term diabetes mellitus, suspicion of primary or secondary amyloidosis (which can be diagnosed by less invasive biopsy), patients with overt malignancy or patients with severe obesity, children under the age of 6 years (because the main cause is minimal change disease), and relapse of steroid-sensitive nephrotic syndrome following the cessation of immunosuppressive

therapy ${ }^{[6]}$.

Since leptospirosis is a condition in which complete recovery of renal function is expected, this diagnosis should be ruled out before a renal biopsy is performed. Consequently, the authors suggest that leptospirosis should be added to the list of nephrotic syndromes where renal biopsy is not indicated.

The prognosis is favourable as the disease generally resolves without permanent sequelae. Advanced age, pulmonary involvement, hyperbilirubinaemia, higher serum creatinine, oliguria, thrombocytopenia and hyperkalaemia are associated with a worse prognosis, with mortality varying from $12 \%$ to $36 \%{ }^{[1]}$. In our case, a complete clinical recovery was observed on the 6th day of antibiotic therapy and the patient's renal functions were normal at 3-week follow-up.

The presentation of leptospirosis as a nephrotic syndrome is rare and only two cases have been described in the literature. Since the recovery of renal function depends on prompt diagnosis and treatment, an accurate and quick diagnostic investigation is warranted because antimicrobial agents can reduce disease severity and duration and lead to a favourable outcome.

\section{REFERENCES}

1. Daher Ede F, Abreu KL, da Silva Junior GB. Leptospirosis-associated acute kidney injury. J Bras Nefrol 2010;32:400-407.

2. Abdulkader RC, Silva MV. The kidney in leptospirosis. Pediatr Nephrol 2008;23:2111-2120.

3. Kshirsagar PP, Sonavane AD, Doshi AC, Teltumbde U, Mandalik S. Atypical presentation of leptospirosis. J Assoc Physicians India 2010;28:114-115.

4. Seyit K, Sezai V, Aytaç K, Osman E, Leyla K, Hamit B et al. A case of leptospirosis presenting with nephrotic syndrome, acute renal failure and thrombocytopenia. Istanbul Tip Fakultesi Dergisi Cilt 2009;2:25-28.

5. Visith S, Kearkiat P. Nephropathy in leptospirosis. J Postgrad Med 2005;51:184-188

6. Becj L, Bomback A, Choi M, Holzman L, Mariani L et al. KDIGO Clinical Practice Guideline for Glomerulonephritis. Am J Kidney Dis 2013;62:403-441. 